\title{
Scanning EM Studies of Normal Human Lens Fibres and Fibres from Nuclear Cataracts
}

\author{
R. J. STIRLING and P. G. GRIFFITHS \\ Newcastle
}

\begin{abstract}
Summary
Normal human lenses obtained from an eye bank, and the central nuclear regions from nuclear sclerotic cataracts, were examined using scanning electron microscopy. Lens fibres were found to possess a varied array of well defined interlocking processes. Fibres from brunescent nuclear cataracts showed complete preservation of membrane architecture in contrast to the extensive membrane disruption that has been seen in cortical cataracts.
\end{abstract}

The vertebrate lens is part of a compound lens system whose function is to focus rays of light onto the retinal photoreceptors. This is accomplished by a population of highly specialised cells, the lens fibres, which are derived from undifferentiated precursors..$^{1,2,3}$ The adult lens must be converging, precisely symmetrical and transmit light in the visible spectrum. It must also have a higher refractive index than the surrounding fluids and yet be flexible enough to focus both near and distant objects.

Previous studies using light and transmission electron microscopy have shown the lens to consist of an anterior epithelium over a mass of lens fibres which have interdigitating interlocking processes. The three dimensional structure of these fibres is best visualised using scanning electron microscopy. However, there has been a paucity of such studies on human lenses partly due to the difficulty of adequate fixation of lens material. The commonly used fixatives penetrate poorly because of the uniquely compact arrangement of the lens fibres.

The aim of this study was to overcome the previously encountered difficulties with fix- ation and examine the three dimensional structure of lens fibres from different depths within the normal lens and compare these with the central fibres of nuclear cataractous lenses.

\section{Materials and Methods}

Six apparently normal human lenses were obtained from the Bristol Eye Bank. Immediately after removal from the donor eye the lenses were placed in Karnovsky's fixative and transported to Newcastle upon Tyne. The ages of the donors ranged from 29 to 69 years of age.

After primary fixation of the intact lens for 24 to 72 hours the lenses were torn into quarters using fine forceps, and these fragments were placed into fresh Karnovskies fixative, for sixteen hours, to ensure complete and even fixation. For studies on cataractous lenses, patients attending for routine cataract extraction were examined preoperatively on a slit lamp, following dilatation of the pupil. Fifteen lenses which showed marked brunescence of the nucleus with a relatively clear cortex were selected for examination. All the patients had visual acuities of less than 6/36.

Correspondence: Dr. R. J. Stirling, Department of Ophthalmology, Newcastle General Hospital, Westgate Road, Newcastle-upon-Tyne 
Diabetics and those with other known ocular pathology were excluded. The ages of the patients ranged from 62 to 69 years of age. Cataract extraction was performed by a standard intercapsular technique. Care was taken to cause minimal disruption of the nucleus which was expressed intact and immediately placed in Karnovskies fixative. An identical two phase fixation process was used as for eye bank lenses. Inevitably some parts of the lenses were crushed during tearing, but these could be easily identified, and avoided during subsequent electron microscopy.

Following fixation the lens material was passed through two changes of $0.2 \mathrm{M}$ sodium cacodylate buffer $\mathrm{pH} 7.3$ each for three hours to wash out all the aldehyde from the lens material.

After dehydration in graded concentations of ethanol and critical point drying, the lens fragments were mounted exposed surface upwards, and sputter coated with gold. The lenses were examined with a Cambridge scanning electron microscope at an accelerating voltage of 10 to $15 \mathrm{kV}$.

\section{Results}

The morphology of lens fibres will be discussed starting from the surface of the lens and working inwards towards the centre. Older fibres are internalised by the addition of younger nerve fibres at the equator this produces an age gradient of fibres within a single lens; the oldest being most deeply situated. The divisions of the lens into cortex and nucleus is somewhat arbitrary as there is continuous gradation in structure rather than the discrete zones of discontinuity apparent on biomicroscopy. In the study the nucleus is defined as the central part of the lens measuring approximately $4.5 \mathrm{~mm}$ in diameter.

\section{Lens epithelium (Fig. 1)}

The epithelium consists of cuboidal cells approximately $5 \mu \mathrm{m}$ thick and $5 \mu \mathrm{m}$ across. These interdigitate to form a flat surface immediately under the capsule, microvilli protrude into the intercellular spaces and may serve to increase the surface area of these cells.

\section{Cortex}

Anterior epithelial cells divide and migrate towards the equator where they elongate to form the most superficial cortex. These young straplike fibres are closely packed with relatively flat surface membranes (Fig. 2). They have not yet developed the surface process or hexagonal shape characteristic of deeper layers. Moving deeper into the lens the mid cortical fibres tend to be thicker and more hexagonal in cross section. Interlocking processes start to appear on the surface of the fibres. There appear to be two populations of interacting joints (Figs 3 and 4). First, fingerlike processes which are seen on the long flat surface of the hexagonal fibres which interdigitate with reciprocal pits on adjacent fibres. Second, flap-like projections coming from the six angular regions formed by adjoining flat surfaces which correspond to depressions on the angular regions of adjacent fibres.

Scanning electron microscopy of the mid cortex demonstrates the regular hexagonal close packing of fibres (Fig. 5).

In the deepest cortical and most superficial nuclear regions the fingers on long flat surfaces are more rounded and the previously flat surfaces are now covered with a fine fingerprint pattern of grooves and ridges. The flap and groove joints on the junctions of the long and the short sides become more rounded and have the appearance of ball and socket joints (Fig. 6). The hexagonal pattern is still apparent though less regular than in the mid cortex.

\section{Nucleus}

The fibres become less regular in shape and the long flat surfaces are thrown into a series of gentle undulations on which the fingerprint pattern is superimposed (Fig. 7). Ball and socket joints are still present but less regular.

The central fibres from lenses with nuclear sclerotic cataracts had identical membrane morphology to those from the clear nuclei of eye bank material in all specimens observed.

\section{Discussion}

This study has confirmed that in common with many other species studied to date $e^{5,6,7,9,10,11}$ the adult human lens contains a varied array of interlocking processes. The nature and arrangement of these fibres is not uniform but varies according to the age and therefore position of the fibre within the lens. 


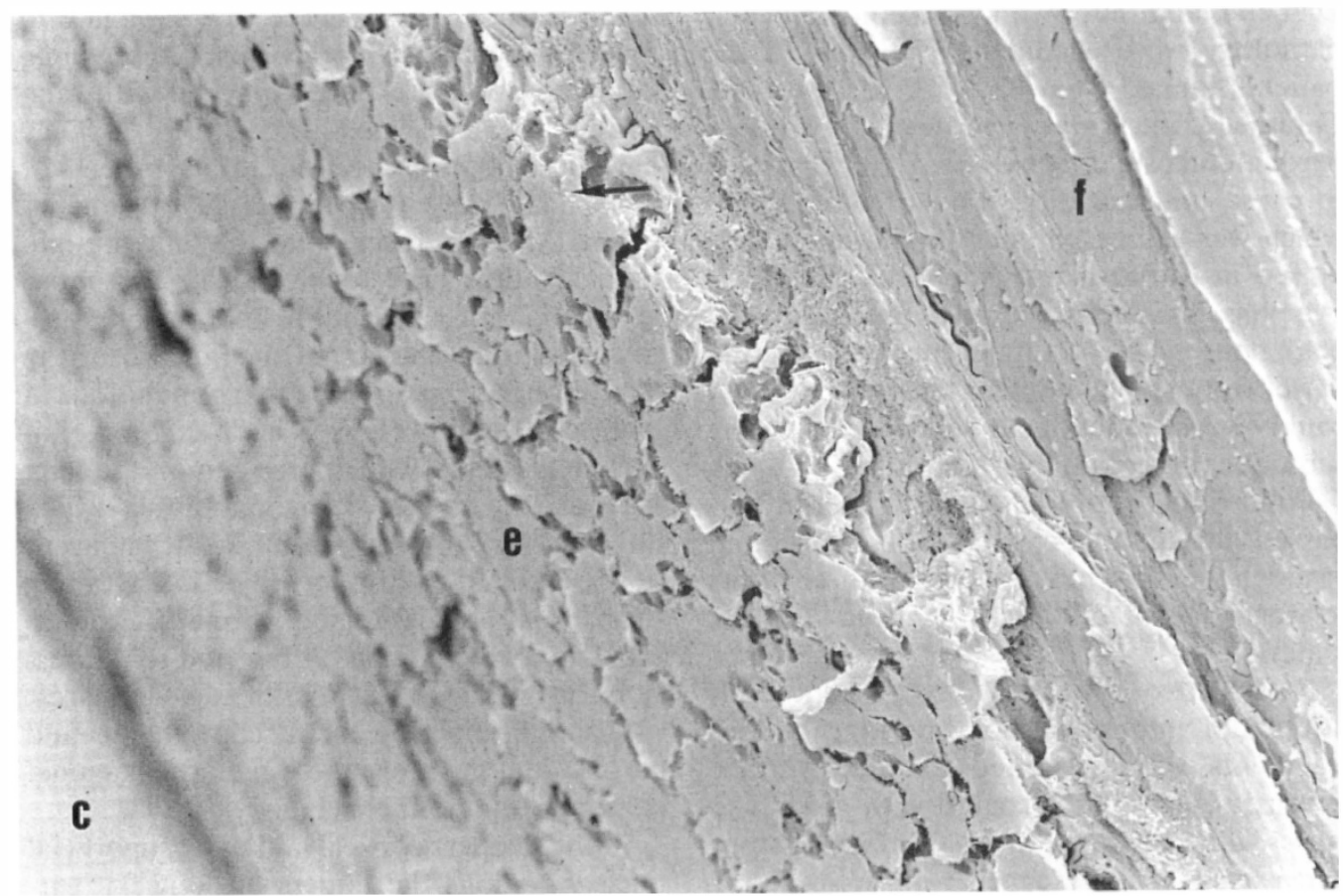

Fig. 1 Scanning EM showing cuboidal epithelial cells (e) with microvilli (arrowed), lens capsule (c) and deeper lens fibres $(f)$.

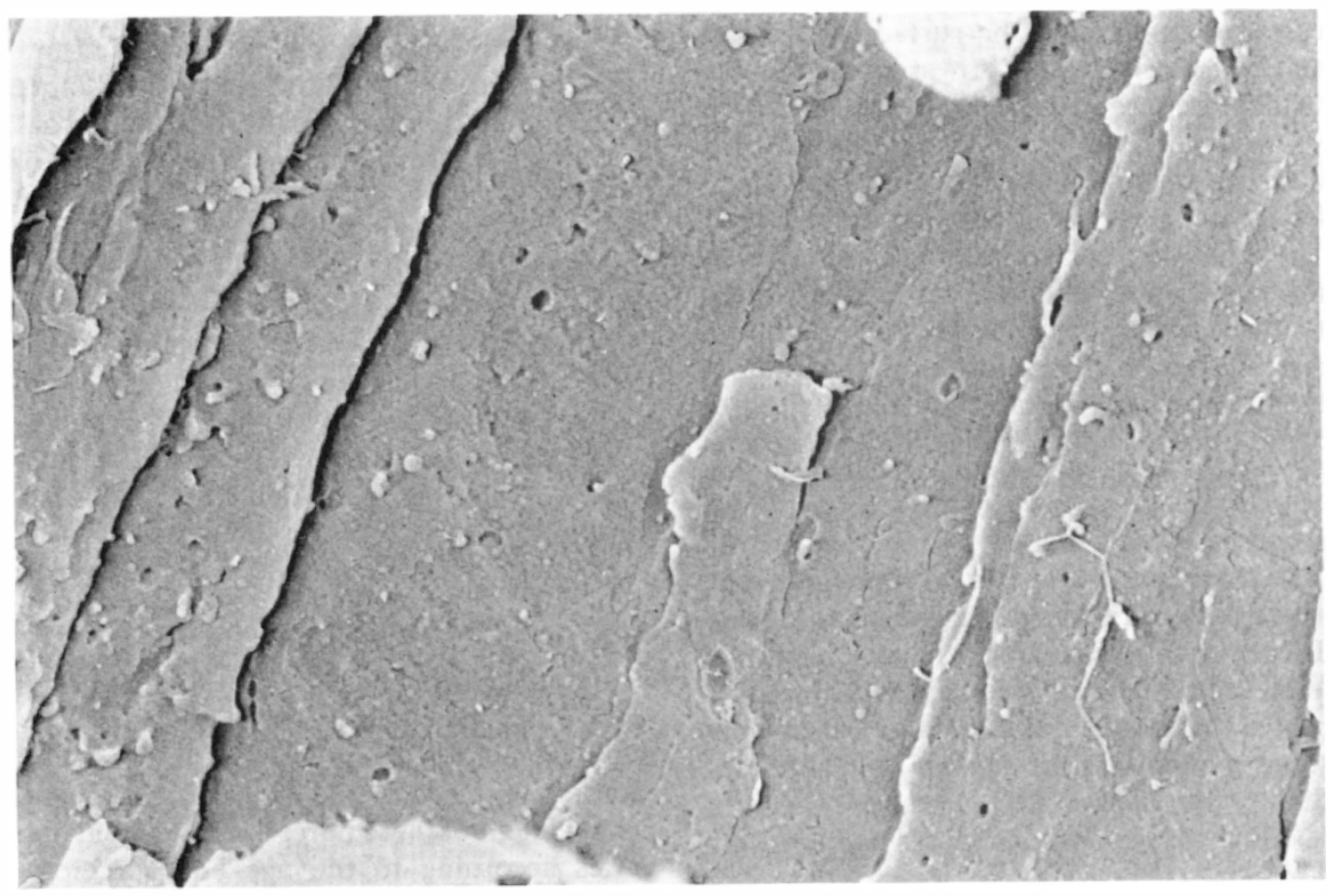

Fig. 2 Scanning EM showing strap like immature cortical fibres with relatively flat surface membranes. 

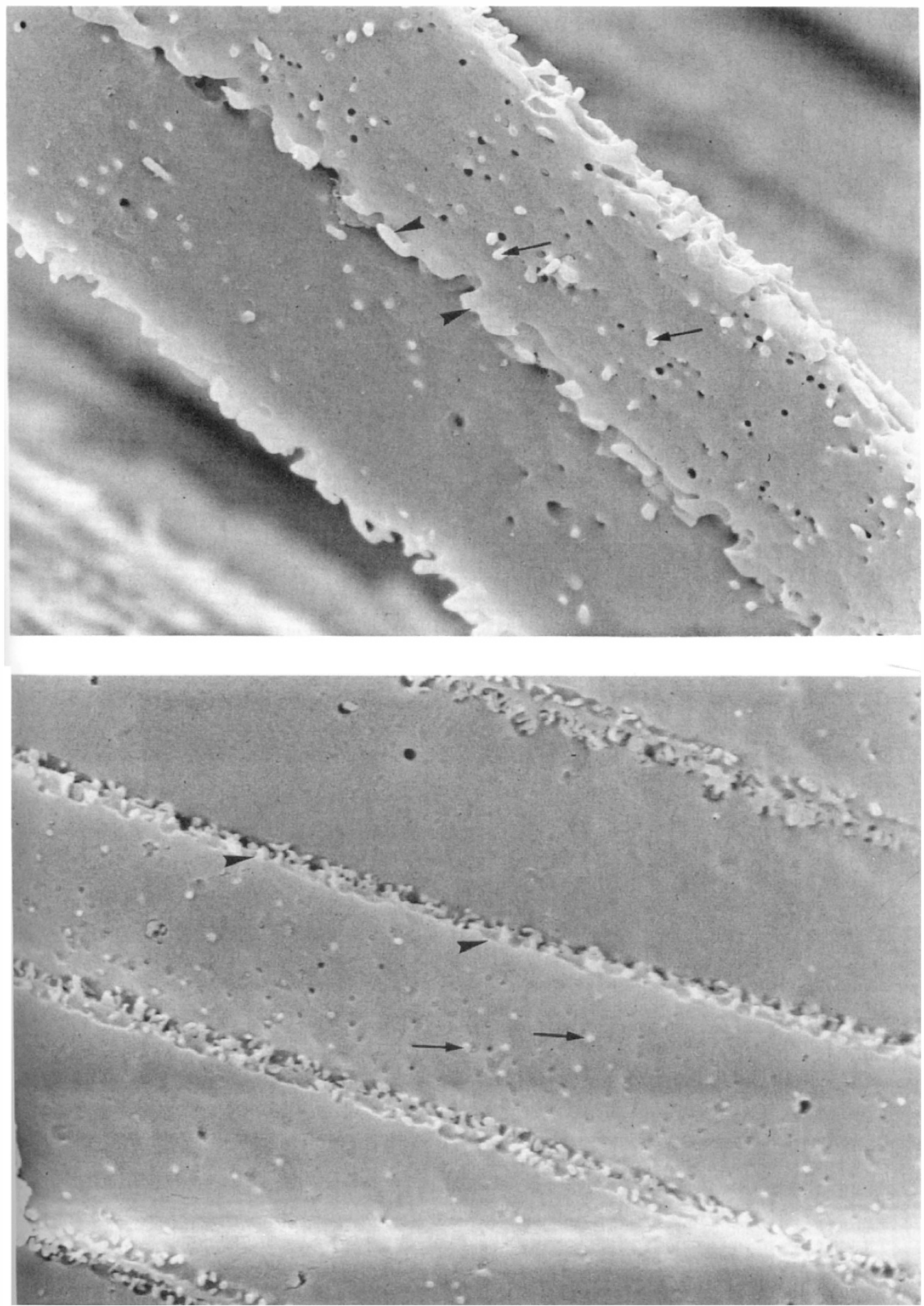

Figs. 3 and 4 Scanning EMs showing mid cortical fibres with fingerlike processes (arrows) on their long flat surfaces and flap like processes (arrow heads) at the angular regions. 


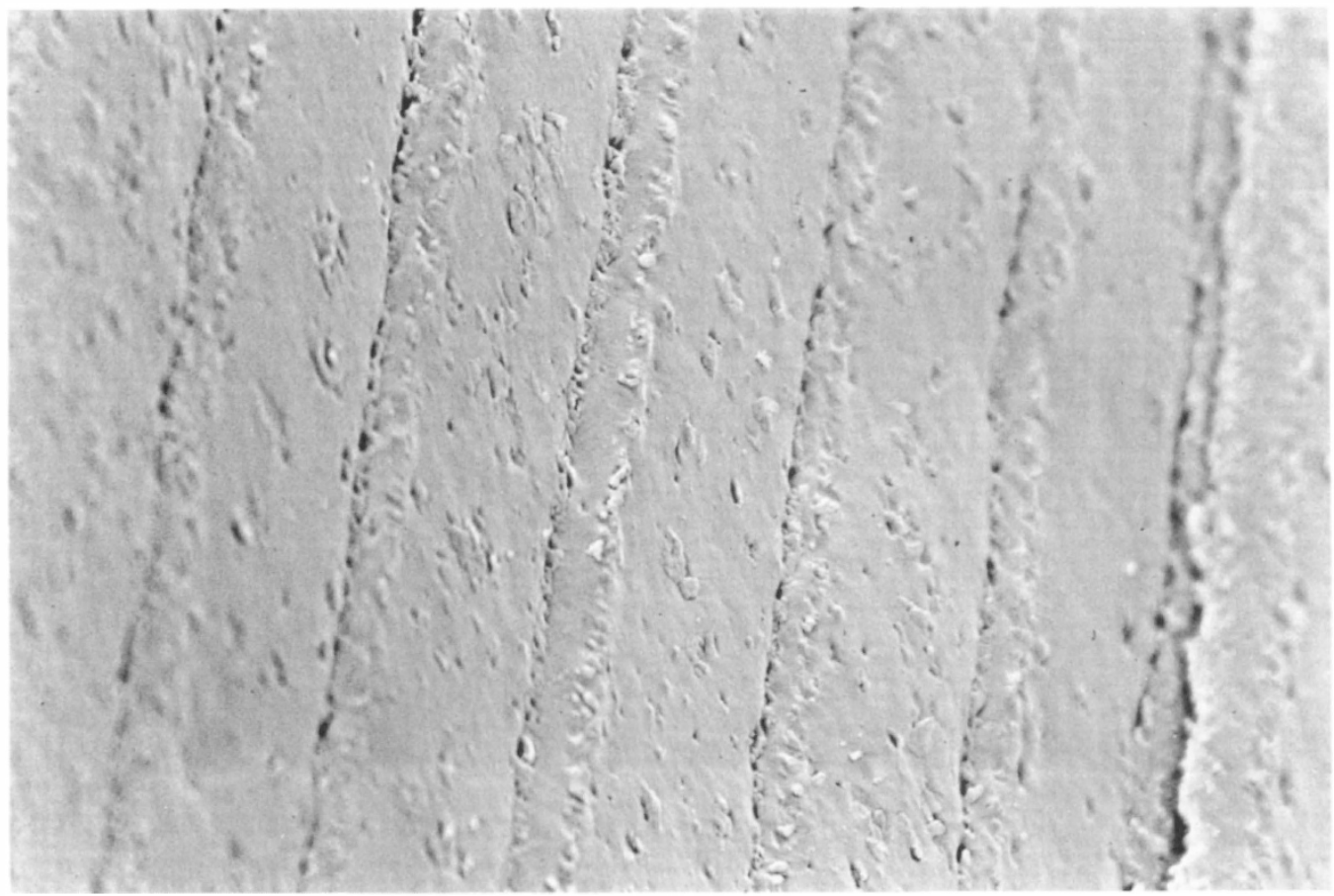

Fig. 5 Scanning EM of mid cortical fibres showing regular hexagonal arrangement.

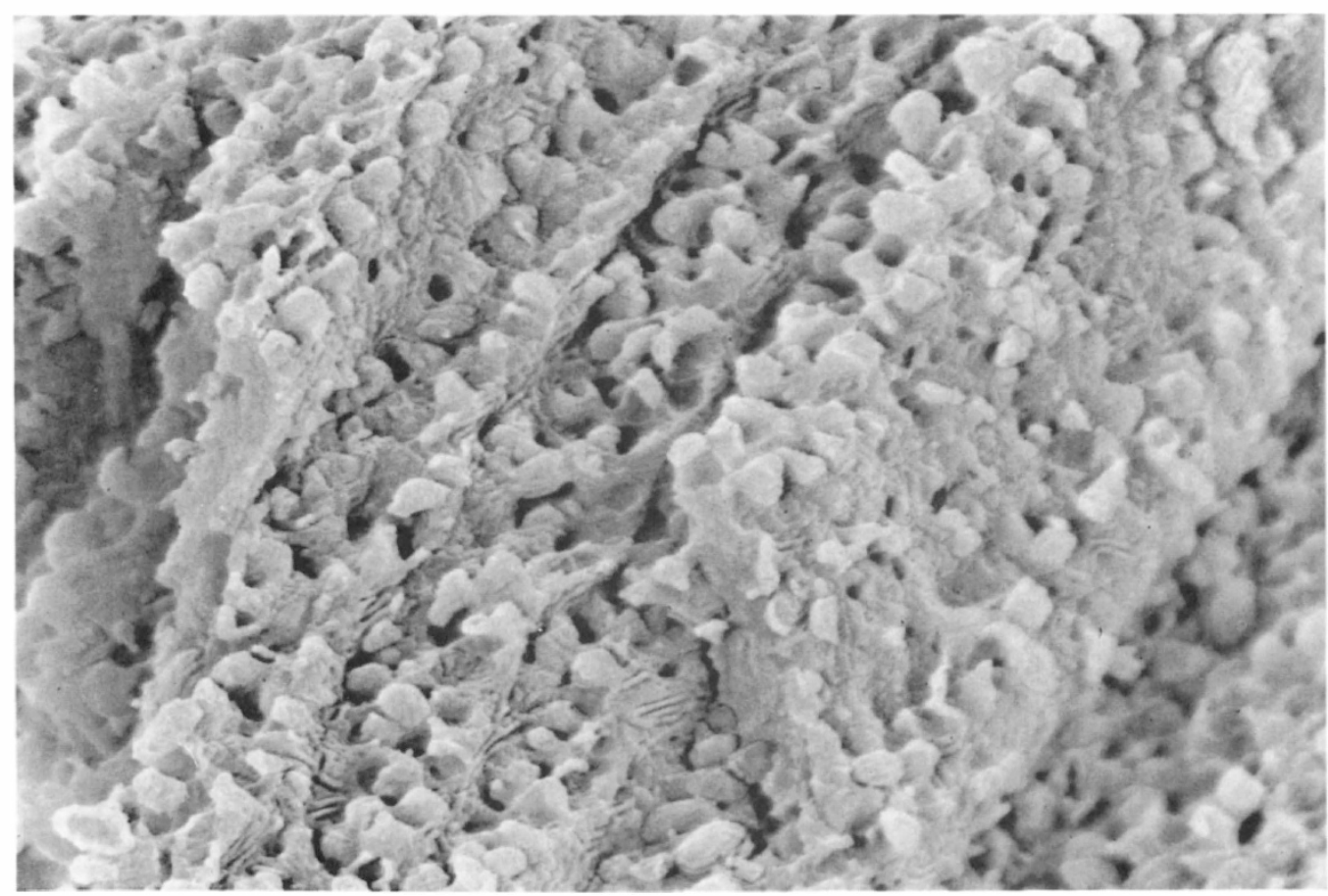

Fig. 6 Scanning EM of deep cortex showing ball (arrow) and socket (arrowhead) joints. The hexagonal shape is just discernable. 


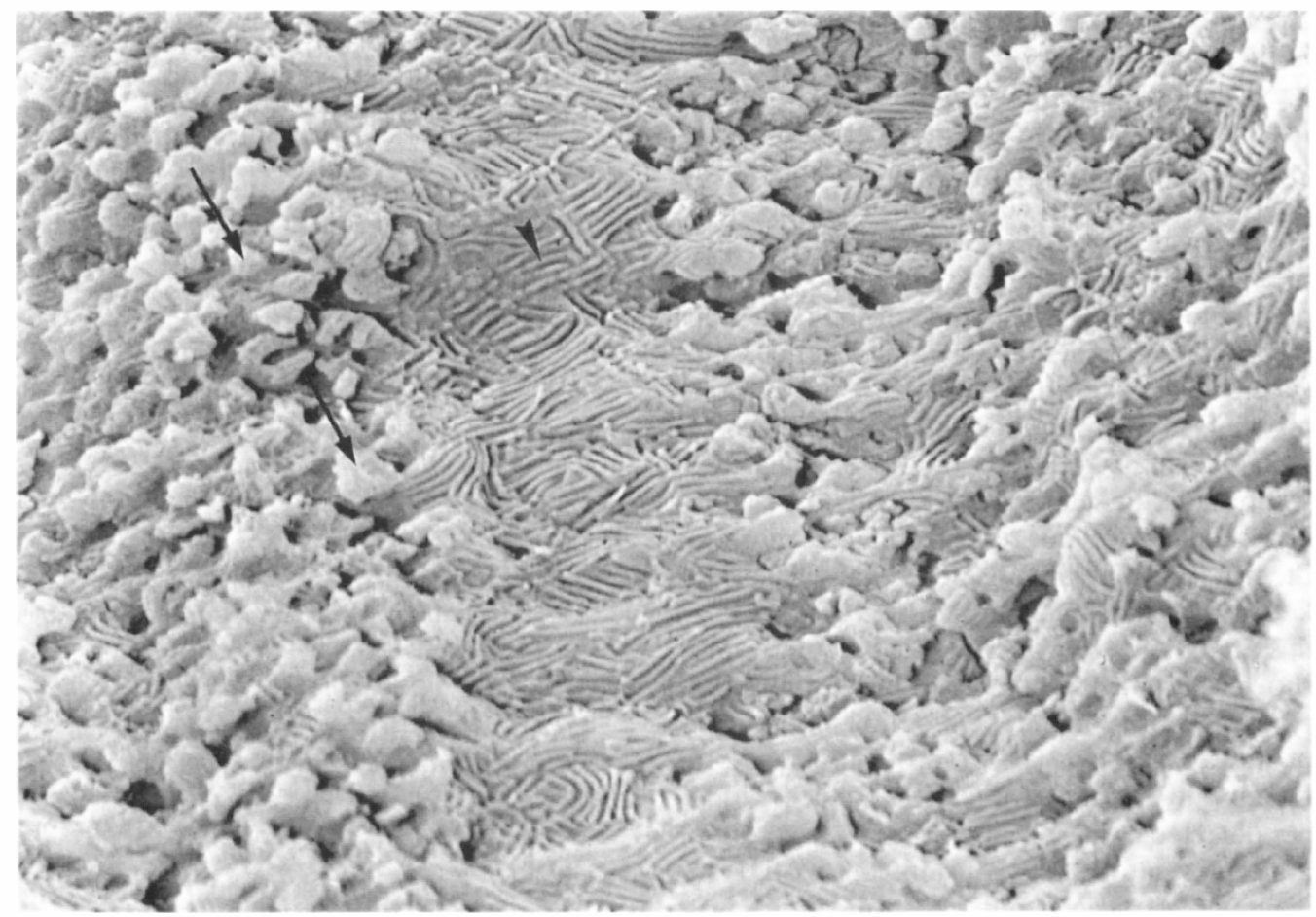

Fig. 7 Scanning EM showing nuclear fibres with gentle undulations on the long flat surfaces with a superimposed fingerprint pattern (arrowhead). Ball and socket joints are still seen (arrows).

The quality of pictures obtained was uniformly good and membrane structure was well preserved at all depths of the lens, showing that the two stage fixation process is adequate. It has been suggested that the paraformaldehyde component of Karnovsky's fixative penetrates tissues feaster than the glutaraldehyde component, temporarily stabilising structures which are subsequently more permanently fixed by glutaraldehyde. ${ }^{4}$ Tearing the lens during fixation, to expose deeper fibres to view, seems to preserve more surface detail than freeze fracture techniques used by some other workers. ${ }^{9}$

For a given amount of zonular tension the lens should assume an exact shape which should be reproducible over countless cycles of contraction and relaxation of the ciliary muscle. Previously a central role has been ascribed to the lens capsule which was said to prevent the lens from assuming its natural discoid shape so that there was a balance of forces between the lens capsule trying to force the lens fibres into a more spherical shape and the elasticity of the lens trying to assume a discoid configuration. In this model, variations in thickness of the lens capsule, lead to the preferential bulging of the anterior pole of the lens. The importance of the capsule in moulding the lens has been challenged and it has been shown that the lens does not assume a flatter discoid shape in the absence of the capsule. ${ }^{9}$ Instead it seems that the lens has some form of tissue memory, such that for a given degree of zonular tension the same shape is adopted, within the constraints set by ageing of the lens.

The prerequisite for elasticity in other materials such as polymers is extensive cross linking so fibres cannot slide relative to one another. The interlocking interdigitating membrane specialisations observed in this study may play a similar role. In particular the tongue and groove joints on the six angular regions of the superficial cortical fibres and the peg and socket joints on the flat surfaces. The more deeply located fibres are held together by ball and socket joints on their angular regions and by interlocking fingerprint patterns on their flat surfaces.

The increased number and irregularity of membrane specialisations may account for the 
structural integrity of the lens nucleus observed at the time of surgery.

Nuclear cataracts appear different to other forms of cataract, instead of appearing white or grey due to scattering of all wavelengths of light, they appear reddish brown suggesting selective absorption of certain wavelengths of light by chromophores. While scattering undoubtedly does occur some of the reduction in vision may be due to absorption of light and spherical aberration. It is therefore not surprising that large scale alterations in structure of individual fibres do not occur. The irregularities responsible for scattering are likely to be at an intracellular level not reflected in alterations in the structure of overlying membranes. This is in keeping with the findings of other workers. Philipson $^{13}$ in a microradiographic study, has shown that nuclear cataracts differ from other types of cataract in that there is no enlargement of intercellular spaces, or breakdown of cell membranes. The only change which could be detected was an aggregation of protein molecules into dense clusters of $50-100 \mathrm{~nm}$ diameter. An SEM study of opaque and transparent states of reversible calf lens cataracts has similarly shown no evidence of large scale irregularity. ${ }^{14}$ Benedek ${ }^{15}$ has theoretically calculated that the presence of albuminoid aggregates with molecular weight of about $5 \times 10 \mathrm{~g}$ $\mathrm{mol}^{-1}$ could in certain conditions explain lens turbidity.

Keywords-cataracts, electron microscopy, lens fibres.

\author{
References \\ ${ }^{1}$ Kuzac J, Alcala J, Maisel H: The surface mor-
}

phology of embryonic and adult chick lens fibre cells. Am J Anat 1980, 159: 395-410.

${ }^{2}$ Torcynski E: Normal and abnormal ocular development in man. Clinical, structural, and Biochemical advances in Hereditary Eye Disorders. 31-51. Pub Alan R Liss Inc., 150 Fifth Avenue, New York. NY 10011.

${ }^{3}$ Stirling RJ and Wakely J. Changes in the surface morphology of lens fibres in the developing chick eye in relation to lens transparency. J Anat 1987, 155: 11-22.

${ }^{4}$ Karnovsky MJ: A formaldehyde-glutaraldehyde fixative of high osmolarity for use in electron microscopy. J Cell Biol 1965, 27: 137-42.

${ }^{5}$ Cohen AI: The electron microscopy of the normal human lens. Invest Ophthalmol 1965, 4: 433-46.

${ }^{6}$ Harding CV, Susan S, Murphy H: Scanning electron microscopy of the adult rabbit lens. Ophthal Res 1971, 8: 443-55.

${ }^{7}$ Oberman AE: Scanning electron microscopy of the lens and zonular fibres. Am J Ophthalmol 1971, 72: 604-7.

${ }^{8}$ Brown NA, Vrensen GI, Shun Shin GA, Willkens B: Lamellar separation in the human lens: The case for fibre folds. A combined in vivo and electron microscopy study. Eye 1989, 3: 597-605.

${ }^{9}$ Jongebloed WL, Figueras, Dilk F, Worst JF: A morphological description of human cataractous lenses by SEM. Doc Ophthalmol 1987, 67: 197-207.

${ }^{10}$ Dickson DH and Crock GW: Interlocking patterns on primate lens fibres. Invest Ophthalmol 1972, 11: 809-15.

${ }^{11}$ Wallenberg M, Wyse J, Lewis B: Surface morphology of lens fibres from eyes of normal and microphthalmic (Bowman) rats. Cell and Tiss Res 1976, 117: 425-38.

${ }^{12}$ Kuwabara T: The maturation of the lens cell: a morphological study. Exp Eye Res 1973, 16: 29-39.

${ }^{13}$ Philipson B: Changes in the lens related to the reduction in transparency. Exp Eye Res 1973, 16: 29-39.

${ }^{14}$ Clark JI, Mengel L, Benedek GB: Scanning electron microscopy of opaque and transparent states of reversible calf lens cataracts. Ophthalmic Res 1980, 12: 16-33.

${ }^{15}$ Benedek GB: Theory of transparency of the eye. Appl Opt 1971, 10: 458-73. 